\title{
Application of digital media and information technology as an educational method in the Ophthalmology journal club
}

\author{
Wasee Tulvatana ${ }^{1}$, Anita Manassakorn ${ }^{2}$, Suppapong Tirakunwichcha ${ }^{3}$, \\ Parima Hirunwiwatkul ${ }^{4}$, Supharat Jariyakosol ${ }^{5}$, Kittisak Kulvichit ${ }^{6}$
}

\begin{abstract}
Background: A journal club session tends to be boring after many hours of work during the week. Attendees lose their attention span shortly after the activity begins.

Objectives: To introduce a new educational model, including the use of digital media devices and social media, in journal club teaching and to evaluate whether this new model could prolong attendees' attention span.

Methods: An online survey from the ophthalmology journal club was conducted to evaluate the range of attention span, the problems and suggestions. Then a team was convened to build a new educational model based on the survey results. Observations in the classroom, the information gathered from the social media, and a post-action online survey were used to evaluate the outcomes. Descriptive and analytical statistics were used for data analyses.

Results: There were 20 journal club sessions during the study period. The journal club is comprised of 30 residents, 10 fellows and 19 staff members. The pre-action survey showed important information about the most boring part and the median attention span was 30 minutes. After the new educational model was introduced, the median attention span increased to 55 minutes $(p<0.001$, Wilcoxon signed ranks test). The status of the attendees, frequency of attendance, and frequency of falling asleep did not show statistically significant association with longer attention span. Students' achievement scores were good.
\end{abstract}

Conclusion: The new educational model increased the attendees' attention span and provided learning achievement in the ophthalmology journal club.

Keywords: Attention, Educational Model, Educational Technology, Internet, Social Media

\section{Introduction}

Not only has a journal club been used as a form of disseminating medical knowledge for a long time but it is also a tool to teach and assess

\footnotetext{
${ }^{1,6} \mathrm{MD}, \mathrm{MSc}$

2, 3, 4, 5 MD

Department of Ophthalmology,

Faculty of Medicine, Chulalongkorn University,

Bangkok, Thailand

Corresponding author:

WaseeTulvatana, MD, MSc

Department of Ophthalmology,

Faculty of Medicine, Chulalongkorn University, Bangkok 10330, Thailand

Email: waseetulvatana@gmail.com
}

\section{Financial support:}

This study was supported by the Classroom Action

Research Fund, Learning Innovation Center,

Chulalongkorn University, Bangkok, Thailand

Trial registration number: TCTR-2011-00023

(www.clinicaltrials.in.th) students' achievement in the critical appraisal skills (Lee et al., 2005; Lee et al., 2006). Most journal clubs begin with presentation of interesting medical articles, then the critical appraisal and the application of the articles. Likewise, at the Department of Ophthalmology, Faculty of Medicine, Chulalongkorn University, we use the journal club to keep members up to date with the most recent articles. The presenter is a first year resident. Everyone will have a chance to critically appraise the articles. Sometimes the presenters may obtain a feedback regarding their presentation skills.

Our department's journal club is normally held at the end of the week, after many hours of work. Journal club attendees seem to lose their attention rather quickly, even though they are aware of the potential benefits. The journal articles were selected by the presenters under supervision of a faculty. Most of the time the selected articles are not distributed beforehand. 
Some articles are distributed in the classroom at the beginning. First year residents would speak in English to summarize the articles and then critically appraise the articles in Thai. It takes 30 minutes to 1 hour to complete the talk. Also, the senior residents would recap and often repeat what had been said. Then the fellows and the faculty members may comment on the papers and the presentation. The session usually ends with the resident's brief reply to the assigned weekly question, which takes about 5 minutes. The total time is approximately 3 hours for 2 articles.

We decided to implement a classroom action research to improve the journal club activities. Firstly, we searched the literature about the journal club improvement method and found that there were reported methods that made the journal clubs more successful: (1) To have a pre-defined meeting schedule, (2) The participants received the articles beforehand, (3) To plan a good meeting, e.g. the exact time slot for the presentation and good food during the meeting, and (4) To have a good conductor during the meeting (Deenadayalan, 2008; Hartzell, 2009). We decided to explore for effective ways to enhance our journal club activities by conducting classroom action research. The objective of the study is to find a new educational model for journal club classes and to evaluate whether this model could increase the attendees' attention span and provide achievement for the residents.

\section{Methods}

The study protocol was discussed, developed and approved by the staff members, fellows and residents of the department before commencement. A confidential online questionnaires was used to gather the information about the attendees' attention span, the most boring part and their suggestions for improvement of the classroom. The journal club team, comprising staff members and residents representatives, set up a new educational model by using the preaction survey results as resource information. The new educational model included (1) creating of a social media group for rapid information distribution, (2) asking the journal club readers/appraisers to set up an online vote for interesting clinical question and/or articles and then select the articles accordingly, (3) using emails, informing all participants of the selected articles at 1 week before the meeting, (4) assigning a master of ceremony to conduct the meeting, (5) preparing nice refreshments to be served during the classes and (6) having the audiovisual system ready for. The conference room was not changed because our department had only one activity room that could accommodate all the staff members, fellows and residents.

After a semester, the participants who were then accustomed to social media voting and the new educational model were asked to reply to the post-action online questionnaires. The attention span was measured and analyzed. In this survey, the most attractive part, the most boring part, and suggestions for improvement of the classes were queried. Achievement of the residents, judged by their scores at the end of semester, was recorded.

Descriptive and analytical statistics including Wilcoxon signed ranks test, chi-square test of potential factors, and multiple logistic regression were used (SPSS version 17.0).

\section{Results}

Our department consisted of 59 persons; 19 instructors, 10 fellows and 30 residents. The first (pre-action) survey results were received from 48 respondents. It showed that the most boring part was the presentation of the first year residents (Table 1 ).

After the survey, the first year residents were instructed to present and discuss the articles in Thai. The senior residents were asked to be brief and straightforward in their comments. The new model was introduced during the study period for a total of 20 journal club classes. Forty articles were selected, $95 \%$ of the articles were from the voting system created in the "journal club group" in the Facebook (Figure 1). All of them were clinical question-based and proposed by the readers/appraisers or other participants.

The attention span was self-reported by the attendees. It was reported that before the new educational model, the median attention span (interquartile range) was 30 (31) minutes, comparing to 55 (35) minutes after the new model introduced $(p<0.001$, Wilcoxon signed ranks test).

We analyzed for factors determining the long attention span, defining as the attention span of 120 minutes or above. Unfortunately, we did not find any significant association between the status of the attendee, the frequency of attendance, and the frequency of falling asleep with the long attention span. 
Table 1: The most boring part in the pre-action period reported by participants via online questionnaires. ( $N=48)$

Answers reported from participants

(Each participant may report more than one item)

First year residents' presentation (In English)

The conference room

Senior residents' discussion

The audiovisual system

Staff's discussion and/or comments

Food and/or drinks

First year residents' discussion of articles (In Thai)

I don't know

I don't care

I never get bored

Others
$\mathbf{N}(\%)$ of total responses

$4(8.3)$

Figure 1: An example of the votes for article in the "journal club group" in Facebook.

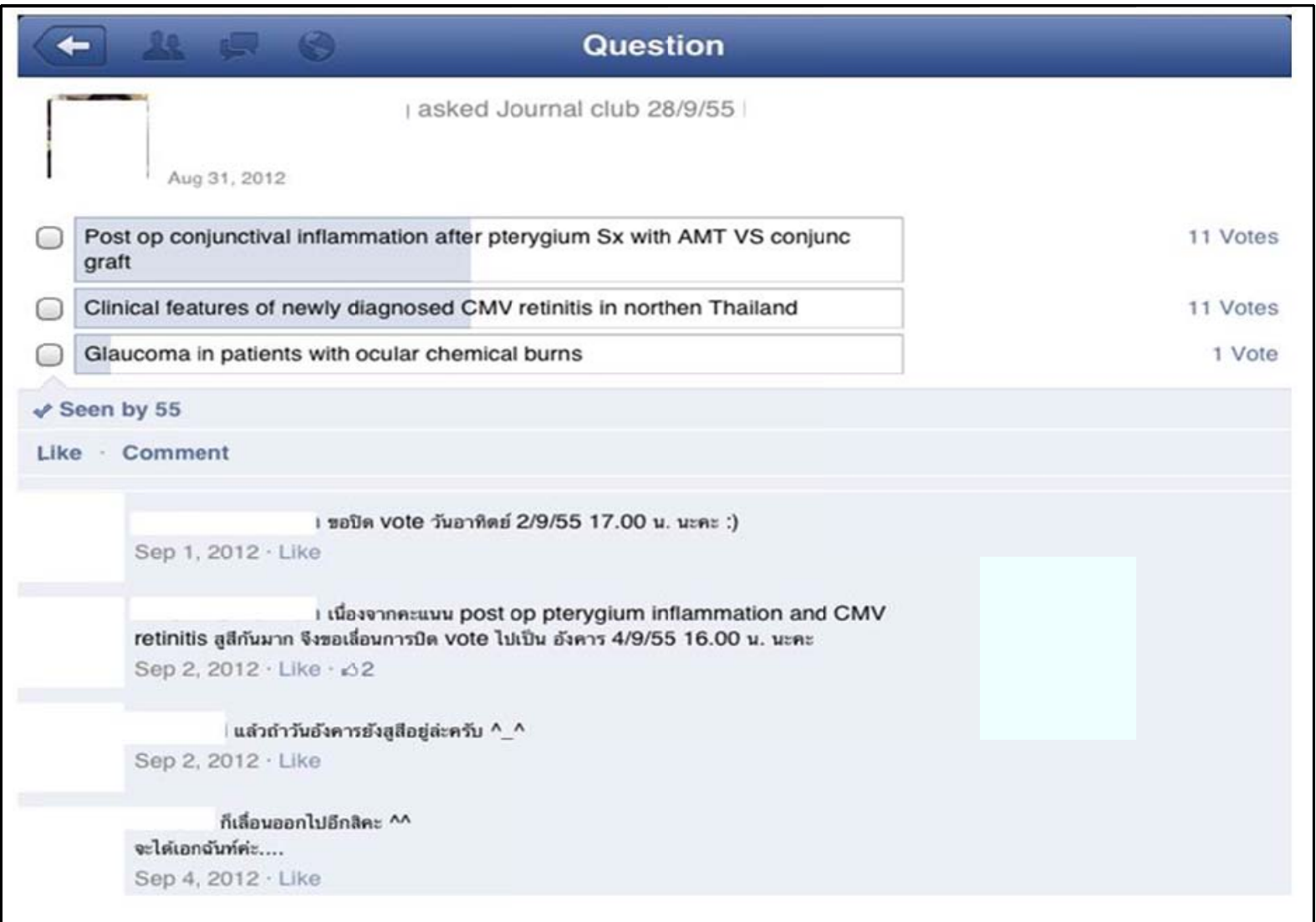


The post-action survey was replied by 42 respondents. The most attractive part of the journal clubs reported in the post-action survey was the staff members comments (Table 2). The most boring part had shifted from the presentation of the first year residents to the senior residents' discussion (Table 3 ). The comparison of the frequency of falling sleep during the class reported by the participants between the pre-action and post-action periods are shown in Figure 2.

Table 2: The most attractive part in the post-action period reported by participants via online questionnaires. $(N=42)$

\begin{tabular}{lc}
\hline $\begin{array}{l}\text { Answers reported from participants } \\
\text { (Each participant may report more than one item) }\end{array}$ & $\mathbf{N}(\%)$ of total responses \\
\hline Staff comments & $35(83.3)$ \\
Weekly question & $23(54.8)$ \\
The articles & $17(40.5)$ \\
Food and/or drinks & $12(28.6)$ \\
First year residents' presentation & $12(28.6)$ \\
Senior residents' discussion & $10(23.8)$ \\
Fellow comments & $7(16.7)$ \\
The audience & $1(2.4)$ \\
The conference room & $0(0)$ \\
Others & $2(4.8)$ \\
\hline
\end{tabular}

Table 3: The most boring part in the post-action period reported by participants via online questionnaires. $(N=42)$

Answers reported from participants

(Each participant may report more than one item)

Senior residents' discussion

First year residents' presentation

The articles

The conference room

The audiovisual system

Unpunctuality

Food and/or drinks

I never get bored

8 (19.0) 
Figure 2: Comparison of the percentage of time fallen asleep reported by participants between the preaction (black boxes, $N=48$ ) and post-action online surveys (white boxes, $N=42$ ).

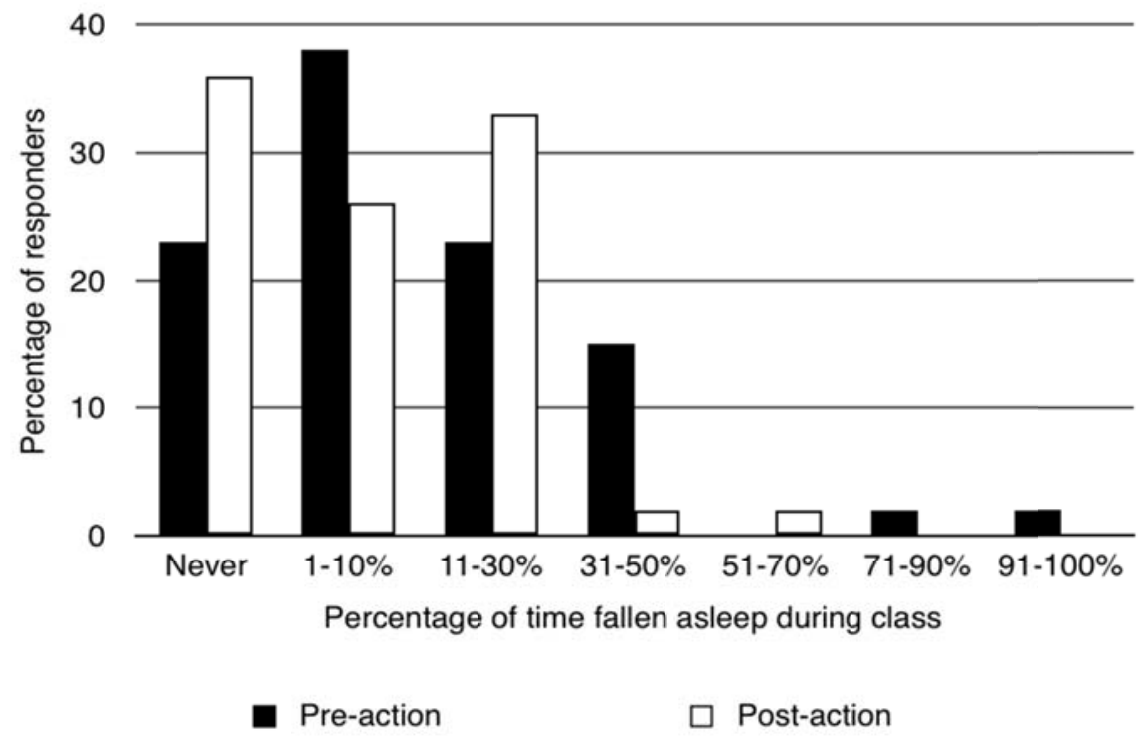

After classes, the discussion continued in the social media in some interesting topics. Interestingly, the activity in the "journal club" group in the social media declined after these journal club sessions end. At the end of the semester, the mean (SD) scores of achievement of the residents were 79.3 $(4.9) \%$.

\section{Discussion}

A new educational model was introduced in the journal club classroom. We found that the digital media and social media had facilitated learning before class, during class and after class. This is to complement the continuous learning attitude for the residents.

Our study has shown that using the new model had increased the attention span of the attendees. The digital media and social media was used to draw more participation and increase the attention span in the new model. We still use face-to-face meeting instead of internet-based meeting since the internet journal clubs were reported to be less effective than moderated journal clubs (McLeod et al., 2010) and collegiality is still as important as the content and critical appraisal (Freshwater, 2011). The food and/or drinks provided for the attendees in our study were not from the drug or device companies as feared by Freshwater
(2011). We intended to preserve our journal clubs to only 3 C's: Collegiality, Content and Criticism. There is no commercialism in our journal club classrooms during the study period.

Although the new educational model has provided satisfactory results, there is still room for improvement. We can study further into the remaining boring part of the classes by the post-action survey. The journal club team would then be possible to modify the activity details as suggested from the study. This is to run the cycle of continuous improvement for the journal club classroom action research. For example, the presentation of the first year residents and the senior residents' discussion still have to be modified. We could not change the conference room, which is rather old, to a new one in a short period of time but this issue should be in the departmental plan. It has to be noted that the effect found in this study might have been from a 'Hawthorne effect' (2012). It would be interesting to see if we can improve the outcome it we went back to the previous educational model. Nevertheless, the new educational model by application of digital media and information technology had improved the attendees' learning attention span and achieved favorable outcomes.

\section{Conclusion}


A new educational model using a combination of digital media, social media, clinical question-based learning, and a good preparation of the activities is proposed. We found that this model has delivered more attention span from attendees in the classrooms and the students accomplish favorable learning achievement. Continuous improvement is needed to run the cycle of classroom action research for this activity.

\section{Acknowledgement}

The study was funded by the Classroom Action Research, Learning Innovation Center, Chulalongkorn University.

\section{References}

Deenadayalan, Y., Grimmer-Somers, K., Prior, M. \& Kumar, S. (2008) How to run an effective journal club: a systematic review, J Eval Clin Pract, 14, pp. 898-911.

Freshwater, M. F. (2011) The four C's for a journal club - ingredients for success or failure, J Plast Reconstr Aesthet Surg, 64, pp. 839-41.
Hartzell, J. D., Veerappan, G. R., Posley, K., Shumway, N. M. \& Durning, S. J. (2009) Resident run journal club: a model based on the adult learning theory, Med Teach, 31, pp. e156-61.

Hawthorne Effect (2012) Wikipedia. [Online]. Available at: http://en.wikipedia.org/wiki/ Hawthorne_effect. [Accessed 31 July 2012].

Lee, A. G., Boldt, H. C., Golnik, K. C., Arnold, A. C., Oetting, T. A., Beaver, H. A., Olson, R. J. \& Carter, K. (2005) Using the Journal Club to teach and assess competence in practicebased learning and improvement: a literature review and recommendation for implementation, Surv Ophthalmol, 50, pp. 542-8.

Lee, A. G., Boldt, H. C., Golnik, K. C., Arnold, A. C., Oetting, T. A., Beaver, H. A., Olson, R. J., Zimmerman, M. B. \& Carter, K. (2006) Structured journal club as a tool to teach and assess resident competence in practice-based learning and improvement, Ophthalmology, 113, pp. 497-500.

McLeod, R. S., MacRae, H. M., McKenzie, M. E., Victor, J. C. \& Brasel, K. J. (2010) A moderated journal club is more effective than an Internet journal club in teaching critical appraisal skills: results of a multicenter randomized controlled trial, J Am Coll Surg, 211, pp. 769-76. 